\title{
The impact of COVID-19 pandemic on pornography habits: a global analysis of Google Trends
}

\author{
Fabio Zattoni $\mathbb{1}^{1} \cdot$ Murat Gül $^{2} \cdot$ Matteo Soligo $^{3}$ - Alessandro Morlacco ${ }^{3}$ - Giovanni Motterle ${ }^{3}$ Jeanlou Collavino ${ }^{3}$. \\ Andrea Celeste Barneschi ${ }^{3} \cdot$ Marco Moschini $^{4} \cdot$ Fabrizio Dal Moro ${ }^{1,3}$
}

Received: 28 July 2020 / Revised: 9 October 2020 / Accepted: 11 November 2020 / Published online: 28 November 2020

(c) The Author(s), under exclusive licence to Springer Nature Limited 2020

\begin{abstract}
As the COVID-19 spread globally, social distancing, self-isolation/quarantine, and national lockdowns have become crucial to control the pandemic. However, these measures may also lead to increases in social isolation, loneliness, and stress, which can alter the consumption of pornography habits. The aim of the study was thus to explore the interest pattern in pornography and coronavirus-themed pornography during the COVID-19 outbreak. Google Trends ${ }^{\circledR}$ was employed to determine the most popular porn websites (Porn, XNXX, PornHub, xVideos, and xHamster), and coronavirus-themed pornography worldwide and in six nations with different COVID-19 outbreak and self-isolation recommendations. We analyzed every search trend on Google ${ }^{\circledR}$ from January 9, 2020 to May 25, 2020 using "joint point regression analysis". Comparisons of week relative search volume (WRSV) and temporal patterns were analyzed to assess the change of interest in search terms during nations lockdowns. Paired $t$-test was used to compare WRSV values among the porn websites during the national lockdowns and the equivalent timespan of the weeks in the previous 4 years. The research trend of almost every keyword increased with significant inflection points for those nations with a straight "stay at home orders" (China, Italy, Spain, and France). "PornHub" and "Porn" showed the highest increase of interest worldwide with an average weekend percentage change (AWPC) of 4.9 and 3.8, respectively. The mean WRSV for keywords in USA and Sweden did not show a similar increase as the other nations. The WRSV percentage change with the historical data had a peak during the straight nations' lockdowns $(p<0.01)$. All the nations had a significant increase in WRSV coronavirus-themed pornography for each keyword $(p<0.01)$ with an AWPC, ranging worldwide between 18.5 and $61.8(p<0.01)$, after the beginning of selfquarantine. As strengths this study uses a big data technology to collect worldwide trend of interest, however, data are anonymous and do not allow analysis of subpopulation groups. In conclusion, we demonstrated an increased interest in pornography and coronavirus-themed pornography after the outbreak of COVID-19 in nations with a straight "stay at home orders".
\end{abstract}

These authors contributed equally: Fabio Zattoni, Murat Gul

Supplementary information The online version of this article (https:// doi.org/10.1038/s41443-020-00380-w) contains supplementary material, which is available to authorized users.

Fabrizio Dal Moro

fabio.zattoni@asuiud.sanita.fvg.it

1 Department of Urology, Department of Medical Area (DAME), University of Udine, Udine, Italy

2 Department of Urology, Selcuk University School of Medicine, Konya, Turkey

3 Department of Surgery, Oncology and GastroenterologyUrology, University of Padua, Padua, Italy

4 Department of Urology, Luzerner Kantonsspital, Spitalstrasse, Luzern, Switzerland

\section{Introduction}

Internet pornography consumption has been increasing due to the widespread use of the internet and become very common practice among people [1]. Today, it has been considered that $46-74 \%$ of men and $16-41 \%$ of women are active pornography users in modern nations [2-4]. These data are supported by one of the most popular porn website, PornHub, as reported over 39 billion searches and 42 billion visits during 2019, suggesting 115 million visits and 18,073 terabytes of data transferred per day [5].

As the coronavirus continues to spread globally, social distancing, self-isolation/quarantine, and national lockdowns have become the cores to control the pandemic [6]. However, these measures may also lead to increases in 
social isolation, loneliness, and stress [7], which can alter the consumption of pornography habits.

This huge amount of pornography consumption can be attributed to have pleasure as a part of masturbation [8]. However, more specifically, the consumption of highvolume pornography is considered to be motivated by several factors [9]. Individual difference variables representing personality traits are emerged as motivators of pornography consumption [10]. The elements of individual difference variables associated with pornography consumption are sensation seeking [11], dispositional sexual affect (erotophobia-erotophilia) [12], and narcissistic traits (entitlement) [13]. In addition to individual difference variables of pornography consumption, selfreported reasons can also be considered as factors that drive individuals to use pornography. Studies reported that sexual arousal and sexual enhancement were the predominant motivations for pornography consumption among the self-reported reasons [14, 15]. Aside from the sexual arousal and enhancement, coping and boredom are linked with greater use of pornography as well $[16,17]$. Studies showed that higher levels of psychological distress often end up with greater levels of pornography consumption [16, 18]. When people have negative feeling like stress or anxiety, viewing pornography could offer temporary relief from those feelings [18]. Similarly, curiosity and information seeking were the other contributing domains for pornography consumption [19]. However, these reasons are less endorsed reasons for pornography use than hedonic reasons [14].

While debates continue on what motivates porn consumption, event-related content searches should also be explored. We know that after a popular event, event-related pornography searches are increasing. For example, during a server crash of Fortnite game, Fortnite-related pornography searches increased [20]. As the coronavirus continues to spread globally, social distancing, self-isolation/quarantine, and national lockdowns have become the cores to control the pandemic [6]. However, these measures may also lead to increases in social isolation, loneliness and stress [7], which can alter the consumption of pornography habits. Concordantly, PornHub reported the first coronavirus search at the end of January 2020, and thereafter coronavirus-themed pornography reached millions of searches [21].

In this context, we hypothesize that this period is giving us the unique opportunity to explore how a pandemic may change pornography consumption on a vast scale. The primary aim of this study is to evaluate the alteration of interest in pornography of affected nations along with possible motivators. This paper also evaluates the tendency toward coronavirus-themed pornography during COVID-19 confinement.

\section{Materials and methods}

In the present study, we used a worldwide search-engine trend, Google Trends $^{\circledR}$ (GT), to determine the societal interest in pornography during COVID-19 outbreak. GT is a website by Google $^{\circledR}$ that analyzes the popularity of top search queries in Google ${ }^{\circledR}$ Search across various regions and languages. It allows to process and analyze "big data" collected worldwide in a certain amount of time. The use of GT in health care research programs is increasing, which provides useful information about epidemiological surveillance, screening, and treatment options [22, 23].

GT generates data and allows the user to compare the relative search volume (RSV) of two or more search terms, offering geographic and temporal models based on the specific terms $[24,25]$. It shows how frequently a given search term is entered into the Google ${ }^{\circledR}$ search engine relative to the site's total search volume over a given period of time. GT can be used for comparative keyword research and to discover event-triggered spikes in keyword search volume. GT also provides keyword-related data including search volume index and geographical information about search engine users.

The RSV is assigned to the search terms. The values of RSV represent the goal of the research based on the highest point of the plot with respect to a region or a specific period. They do not represent absolute search volume numbers since the data are presented on a scale from 0 to 100 . The value of 100 indicates the highest frequency of the searched term, while 50 indicates half of it. The 0 score, however, indicates that no sufficient data were found regarding the search term $[22,23,26]$.

\section{Data selection}

Three volunteers (two men and one woman) aged between 25 and 45 who are heterosexual and do not use porn frequently ended up with 63 pornography websites after a website search (Appendix 1). All the website names were queried in GT. Multiple comparisons were performed until we found the most popular five websites in the last 4 years worldwide.

\section{Data collecting for interest in porn websites}

Five porn websites (Porn, XNXX, PornHub, xVideos, and xHamster) were included in the present study as the most popular porn websites according to GT. We queried GT and downloaded the data search input for all the words listed above all at once for the last 6 months (January 9, 2020 to May 25, 2020). We extracted data for six nations that were affected differently by the COVID-19 outbreak: China as the first nation to experience the COVID-19 
infection, Italy Spain, and France as major COVID-19 outbreaks in Europe with a very straight national order to stay at home, Sweden as a nation in Europe without confinement measures, and finally, we analyzed the United States (USA) as one of the worse affected countries with the localized lockdown. We performed analysis from January 9, 2020 since that day China makes public the genome of the coronavirus, proving its link to SARS and MERS viruses. Scientists were then able to develop diagnostic tests for viral genetic material from nose and throat swabs. We superimposed the new cases/day curve of COVID-19 for each country with an interest in porn websites for each nation.

\section{Data collecting for comparison of interest in porn websites} with the preceding 4 years

After extracting the above-mentioned data, the same data were downloaded for the equivalent timespan in the previous 4 years according to the weekdays. This was done to assess the percentage change compared with the equivalent timespan of the previous 4 years.

\section{Data collecting for coronavirus-themed pornography and paraphilic}

To evaluate the coronavirus-themed pornography, we downloaded the data search input all of the following data input at once: "coronavirus + porn," "covid + porn," "coronavirus + sex," "covid + sex," "coronavirus + pornography." We also searched the terms like "xibitionism," "zoophilia," "necrophilia," "coprophilia," "urophilia," "klismaphilia," and "mask" to evaluated the change in interest in paraphilic themes. The data were downloaded from January 9, 2020 to May 25, 2020 within the same geographic areas.

\section{Raw data from pornography websites}

The five pornography websites included in the study were contacted by e-mail to use their official raw data for performing further analysis.

\section{Data analysis and statistics}

Since data are fluctuating overtime, we decided to use the mean weekly RSV (WRSV). The WRSV is calculated as mean relative daily search volume within the same week. The join-point regression (JPR) [27] model was used to define significant changes in mean WRSV over time for each term search. The JPR model is used to better describe trends that are not persistent over the time and allows for evaluating statistically significant changes (join points) in trends. If it is determined that the week(s) in which the changes (junction points) occurred in the trend were statistically significant, the regression parameters can be estimated by linear regression methods [27, 28]. For this reason, segmental periods are specific for each search [26].

Linear trends in RSV were summarized using the estimated WRSV and week percentage change (WPC). WPC was used to measure differences in WRSV between two join points. Average weekend percentage change (AWPC) and the respective $95 \%$ confidence intervals (CIs) were estimated to summarize linear trends in WRSV during the entire period [27, 28]. WPC was significantly different from zero at the alpha $=0.05$ level.

The paired $t$-test was used to compare WRSV values among the five selected porn websites during the national lockdowns (January 9, 2020 to April 9, 2020) and the equivalent timespan of the weeks in the previous 4 years. A two-tailed test with $p<0.05$ was considered statistically significant. All the statistical analyses were performed
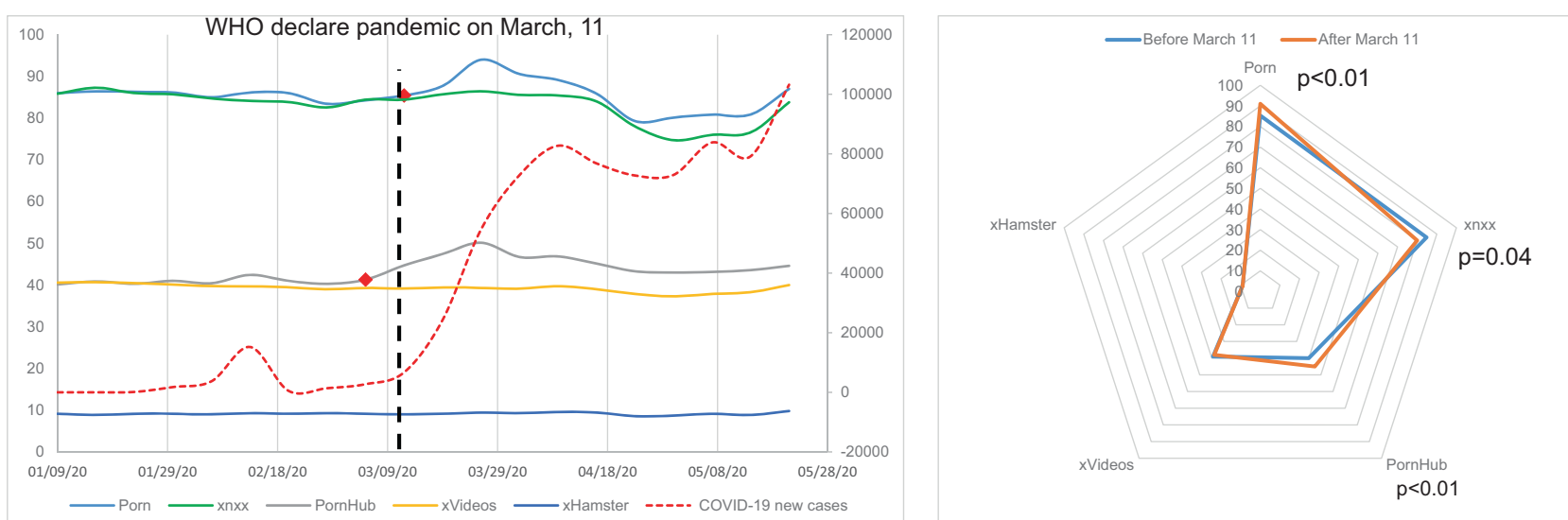

Fig. 1 Week relative search volume (WRSV) for "Porn," "XNXX," "PornHub", "xVideos", "xHamster" for the entire world over time. Significant changes in slope within the national lockdown are highlighted with a red rhombus. Red line is the superimposed new COVID-19 cases (color figure online). 


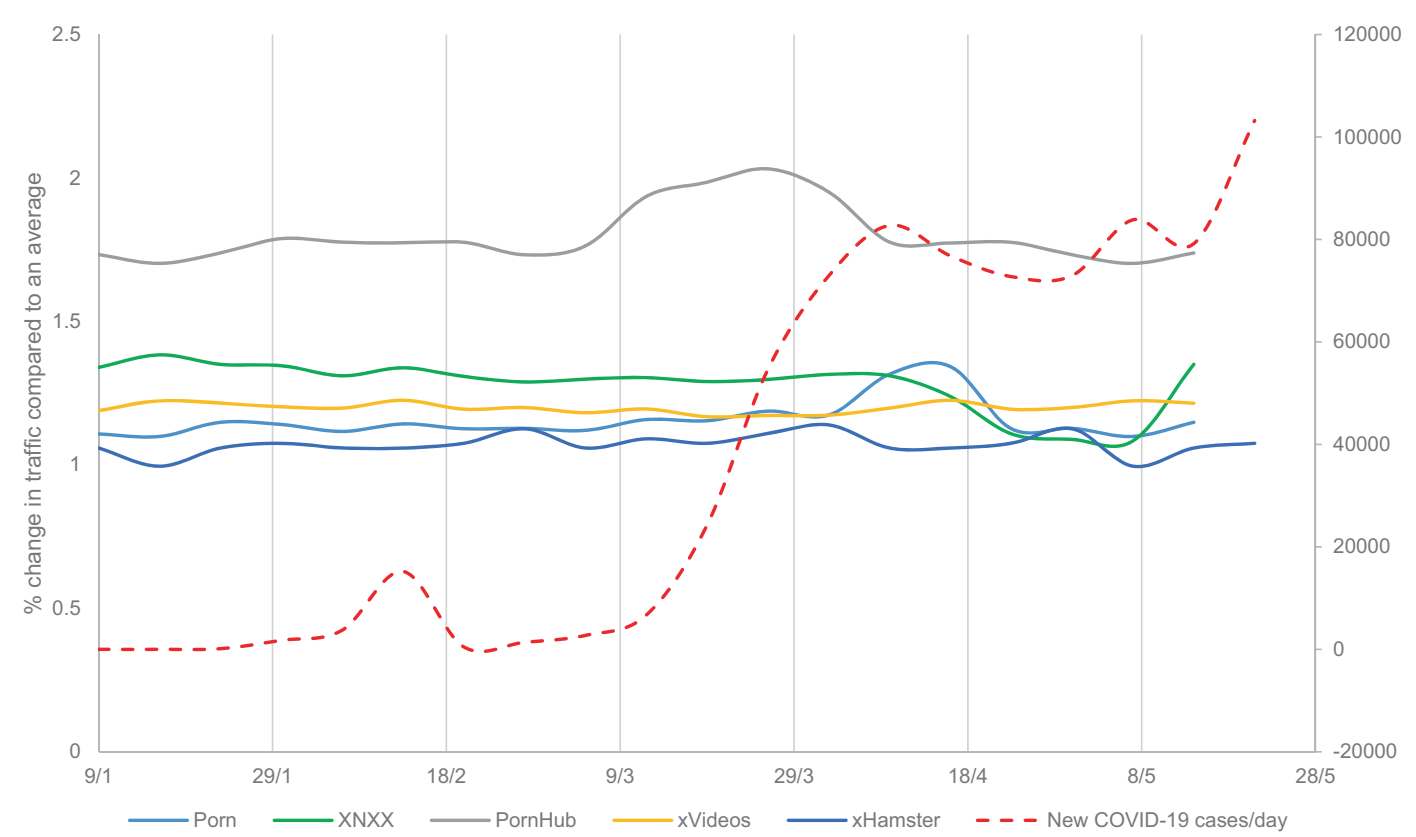

Fig. 2 Week relative search volume (WRSV) percentage change compared to the mean WRSV of the equivalent timespan of previous 4 years for the entire world over time. The red line is the

using SPSS v.24.0 (SPSS Inc., Chicago, IL, USA) and Join Point Trend Analysis Software V. 4.2.0.2 (Statistical Research and Applications Branch, National Cancer Institute, Bethesda, MD, USA). Appendix 2 summarizes the assumed lockdown dates for countries evaluated in this study.

\section{Results}

\section{Change of interest in porn websites for nations with the worse COVID-19 outbreak}

The WRSV over the time for the five keywords, respectively, for China, Italy, Spain, France, USA, Sweden, and the entire world is demonstrated in Fig. 1 and Supplementary Fig. 1a-f. Overall, there is an increased trend of interest in pornography website research on Google ${ }^{\circledR}$ for "PornHub" (AWPC: 4.9, 95\% CI: 2.1; 57.1; $p=0.04$ ), "XNXX" (AWPC: 1.8 , 95\% CI: 0.6; 3.8; $p=0.03$ ), and "Porn" (AWPC: 3.8 , 95\% CI: $1.9 ; 5.7 ; p=0.04$ ) after the WHO declared COVID-19 pandemic.

China had an exponential increase in WRSV at the beginning of January 2020 for "xVideos" (AWPC: 10.4, 95\% CI: $2.4 ; 20.1 ; p=0.05$ ), "Porn" (AWPC: $22.2,95 \%$ CI: $1.2 ; 79.7 ; p=0.03$ ), and "PornHub" (AWPC: 72.7, 95\% CI: $50.1 ; 101.2 ; p<0.01)$, respectively. The highest peak was reached at the end of January 2020, after that, a significant decrease was found for "xVideos" (AWPC: -12.4 , 95\% CI: $-28.2 ;-6.8 ; p=0,01$ ), "Porn" (AWPC: -5.2 , superimposed new COVID-19 cases during the corresponding months of 2020 (color figure online).

95\% CI: $-12.6 ;-1.2 ; p=0.02$ ), and "PornHub" (AWPC $-9.9,95 \%$ CI $-13.3 ; 6.4 ; p<0.01)$, respectively.

Italy had an increased WRSV after the national lockdown at the beginning of March 2020 for "Porn" (AWPC: 1.9, 95\% CI: $1.1 ; 13.4 ; p=0.04$ ), "PornHub" (AWPC: 12.1, 95\% CI: 9.2; 57.7; $p=0.04$ ), Xvideo (AWPC: 3.2, 95\% CI: $1.1 ; 12.2 ; p=0.05$ ), and XNXX (AWPC: $4.1,95 \%$ CI: $5.9 ; 27.7 ; p=0.03)$, respectively. France had a significant increase of WRSV for "PornHub" (AWPC: 17.8, 95\% CI: 12.1-21.9; $p=0.01$ ) and "Porn" (AWPC: 5.6, $95 \%$ CI: $0.9 ; 10.1 ; p=0.01)$ at the beginning of March 2020.

For Spain, the WRSV for "PornHub" increased after the national lockdown touching a higher rate in march 2020 (AWPC: $11.6,95 \%$ CI: 9.8; 45.0; $p=0.04$ ). The mean WRSV for keywords in USA and Sweden did not show a similar increase as the other nations.

\section{Percentage change compared to the equivalent timespan of the previous 4 years}

Figure 2 and Supplementary Fig. 2a-f depict the WRSV percentage change compared to the mean WRSV of the equivalent timespan of the previous 4 years. The percentage change of "PornHub" overtime increased, respectively, in China, Italy, Spain, and France $(p<0.01)$. Interestingly, a peak of percentage change was found during the national lockdowns around the day with a major point of new COVID-19 case/day. Neither in USA nor in Sweden a significant different in percentage change was observed. 
Fig. 3 Week relative search volume (WRSV) of coronavirus-themed pornography for the entire world over time. Significant changes in slope within the national lockdown is highlighted with a red rhombus. Fetishism is used as a comparative terms of paraphilic pornography. Red line is the superimposed new COVID-19 cases (color figure online).

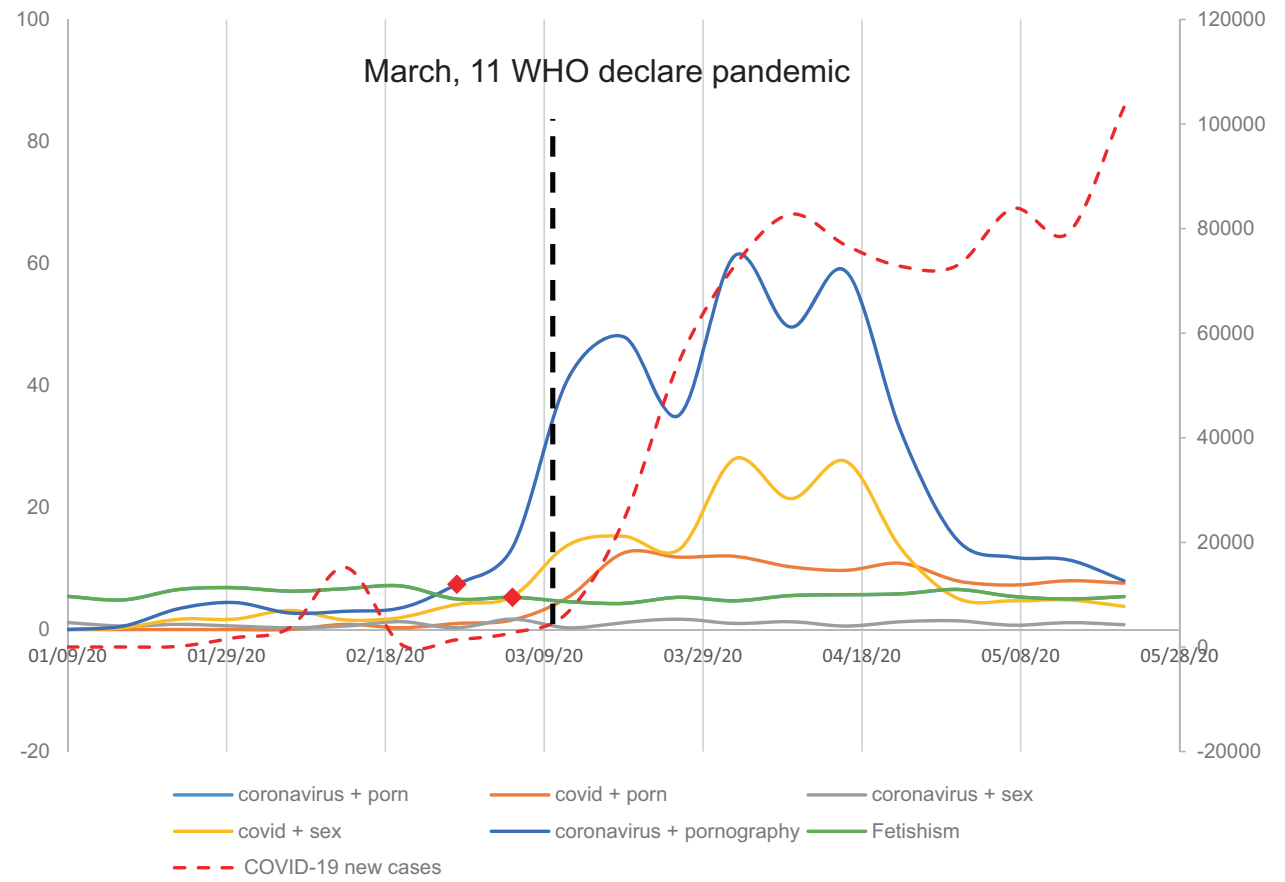

\section{Coronavirus-themed pornography}

Figure 3 and Supplementary Fig. 3a-f depict the coronavirus-themed pornography. Overall, there was a significant increase in WRSV coronavirus-themed pornography for each keyword $(p<0.01)$ around the day with a major point of new COVID-19 case/day. In particular, we found an AWPC after the lockdown ranging between 18.5 and 61.8 worldwide $(p<0.01)$.

We did not find any change during the last 4 years for "fetishism," "transvestism," "exhibitionism," "zoophilia," "necrophilia," "coprophilia," "urophilia," "klismaphilia," and "mask." Since fetishism was the most popular, we included it in the analysis as a comparison to the COVID19-related terms.

\section{Data from PornHub}

Of the five pornography websites queried for raw data, only PornHub allowed us to download their statistical analyses. The internet traffic percentage change during COVID-19 pandemic and its stratification by sex and age groups is shown by Fig. 4a-d. Data show that in late March 2020 a $25 \%$ increase of total traffic change was compared to an average day in the past 4 years in both sexes and age groups.

PornHub website also confirmed a worldwide increase in coronavirus-related search porn (searches containing "Corona" or "Covid") during the lockdown, which means people also intend to watch more coronavirus-themed porn [21].

\section{Discussion}

Across the globe, the coronavirus pandemic is affecting almost all aspects of daily life. To limit the spread of COVID-19 pandemic, lockdowns have been instituted in major cities and countries around the world. For this reason, people stayed isolated or self-quarantined at homes to prevent the spread of the virus and to avoid becoming infected. According to our analyses, we showed that people are finding creative ways to spend the time like watching porn at home. A sharp increase in porn searches was seen in nations where coronavirus is widespread. One of the most popular porn website reported that their traffic has steadily increased in March as the pandemic has spread [21], which confirms our findings. Interestingly, there was no different WRSV in nations like USA and Sweden, where regulations were never as restrictive as the ones adopted in Italy, Spain, and France.

Boredom is also considered a possible trigger of hypersexual behavior by Kafka [29]. This notion is supported by a psychology research demonstrating that leisure boredom is a significant predictor of pornography use, suggesting that people consume porn more when they are bored [30]. Rothman et al. [31] define the use of pornography as a tool to relieve boredom. Similarly, a recent systemic review evaluating the association between boredom and hypersexuality identified a link between boredom and increased online sexual activity [32]. The rationale behind this boredom effect may be due to the men's novelty-seeking behaviors to reduce monotony and increase arousal [33]. 

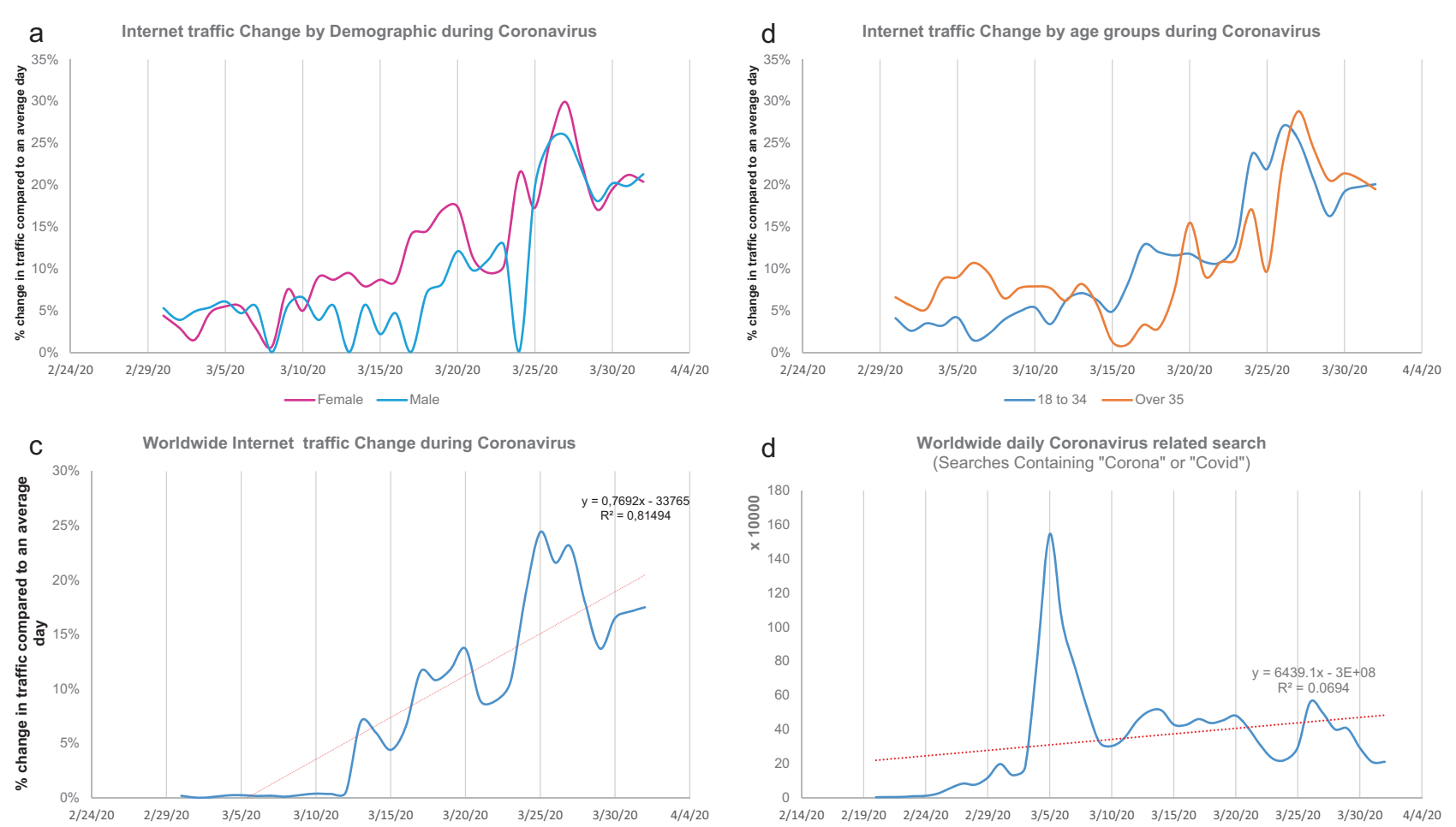

Fig. 4 The internet traffic percentage changes according to "PornHub" website compared to an average day in the past 4 years. An increase in internet traffic search is shown for each gender (a), age groups (b), worldwide (c), and coronavirus-themed search (d).

Another reason for increased porn consuming could be that some people are using sex as a surviving mechanism for coping with their loneliness, depressive symptoms, and even fear of death [34-36]. In a study, Baltazar et al. [15] reported that people are endorsing porn use to cope with negative affect. Again, in a study, pornography consumption is an important tool for mood management and stress relief [16]. It should be noted that problematic pornography consumption is also considered to cope with negative emotions [18]. As described by Lehmiller [34], the key idea behind Terror management theory is that "when we are reminded of our own mortality, we subconsciously alter our attitudes and behaviors to help us cope with the 'terrifying' prospect of our eventual death." A research demonstrated that when we are faced with the prospect of our own mortality, this prompts sexual desire and behavior as a coping mechanism [37]. To the extent that the COVID-19 pandemic is making mortality more salient, it would make sense that a person would feel a rise in lust, which could partly explain why more porn is being consumed. Interestingly, some survey studies investigated sexual function during restrictive social distancing and found how risk perception of COVID-19 was negatively associated with frequencies of sexual activities [38-40]. In particular, a higher general anxiety during COVID-19 was showed to have a negative impact on sex life and frequencies of sexual intercourse, decreased satisfaction with sex life and frequencies of sexual activity [41]. These results could explain the use of pornography as an escape from the reality and sexual life crisis.

The interest in coronavirus-themed porn has also increased during this pandemic in all nations. In March 2020, more than 1.8 million coronavirus searches have appeared on Pornhub [21]. Coronavirus-themed porn mostly includes sex with masks, surgical gloves, and hazmat suits. The reason behind the search of this kind of may be related to people's constant need for sexual novelty and humans' ability to fetishize virtually everything [42]. On the other hand, another explanation of watching coronavirus-themed porn might be an eroticization of fear. It has been well established that strong emotions are often mistaken for sexual attraction [43]. For example, when people engage in activities that produce high-arousal states-like riding a roller coaster-and then encounter an attractive stranger, attraction to that person increases. High-fear states have the potential to amplify sexual arousal and attraction. In this context, if people are on edge from coronavirus news (meaning they are in a heightened state of generalized physiological arousal) and see a media image of an attractive person wearing a mask, this could lay the foundation for them to start sexualizing coronavirus imagery.

Several inherited limitations to the current study need to be acknowledged. First, the data obtained by GT are 
anonymous and do not allow the analysis of subpopulation groups. It is important to note that the 100 reference RSV value is for the time when the search term had the highest volume "relative to all Internet searches at the same time" and subsequent value of 50 does not represent half as many gross searches as the 100 time. In addition, some barriers like, language, internet access to porn websites, or religious restrictions for some other nations might have compromised our search results. Our results showed that "PornHub" is the most popular porn website during the lockdown, but it might have coincided with the free premium account access to encourage people to stay indoors and distance themselves socially. With the available data, we cannot disentangle whether people consumed more pornography during the lockdown due to lockdown measures or free access to premium porn content. However, major pornography websites always present free videos and do not require any payments for basic access. Besides, in some nations (e.g., Italy) after the end of the free premium account on April 23, the interest for porn websites was still higher than before the lockdown. Moreover, although some porn websites did not offer a free premium account, there was an increased interest in pornography anyhow. Again, some nations (e.g., China) had an increased interest in pornography even if PornHub did not offer a premium account. Last but not least, increased interest in pornography was observed even before the date Pornhub offered a free premium account for Italy on March 12 and for Spain and France on March 16. Unfortunately, GT does not allow an evaluation of the network address of users and the "time using" of porn. Thus, we cannot exclude that the same person was watching more porn or there were different users. Another limitation is that due to the dynamic nature of internet, research volumes may be fluctuating over time. As a GT limitation, we were not able to perform sub-analysis of porn categories. Finally, we assumed that people are accessing porn websites as typing the name of the intended website into Google ${ }^{\circledR}$ search bar. But actually, many others may directly access the intended porn website by directly typing the name of the website or hitting the bookmarks, which may strengthen our results.

\section{Conclusions}

The COVID-19 outbreak has affected the use of pornography and its consumption. After national order of selfrestriction, we demonstrated that there was an increasing search trend of online porn and coronavirus-themed pornography. Further psychological research and sexual behavior analysis are needed to assess the impact of a social crisis such as a pandemic on pornography consumption habits.

\section{Compliance with ethical standards}

Conflict of interest The authors declare that they have no conflict of interest.

Publisher's note Springer Nature remains neutral with regard to jurisdictional claims in published maps and institutional affiliations.

\section{References}

1. Luscombe B. Porn and the threat to virility. The first generation of men who grew up with unlimited online porn sound the alarm. Time. 2016;187:40-7.

2. Rissel C, Richters J, de Visser RO, McKee A, Yeung A, Caruana T. A profile of pornography users in Australia: findings from the Second Australian Study of Health and Relationships. J Sex Res. 2017;54:227-40.

3. Lewczuk K, Gola M. Changes in Internet pornography use between 2004 and 2016: a study of a representative sample of the Polish population. J Behav Addict. 2018:104-04.

4. Regnerus M, Gordon D, Price J. Documenting pornography use in america: a comparative analysis of methodological approaches. J Sex Res. 2016;53:873-81.

5. Pornhub. The 2019 year in review. https://www.pornhub.com/ insights/2019-year-in-review (2019).

6. Zhang Y, Ma ZF. Impact of the COVID-19 pandemic on mental health and quality of life among local residents in liaoning province, China: a cross-sectional study. Int J Environ Res Public Health. 2020;17:2381. https://doi.org/10.3390/ijerph17072381.

7. Wang C, Pan R, Wan X, Tan Y, Xu L, Ho CS, et al. Immediate psychological responses and associated factors during the initial stage of the 2019 coronavirus disease (COVID-19) epidemic among the general population in China. Int J Environ Res Public Health. 2020;17:1729. https://doi.org/10.3390/ijerph17051729.

8. Solano I, Eaton NR, O'Leary KD. Pornography consumption, modality and function in a large internet sample. J Sex Res. 2020;57:92-103.

9. Grubbs JB, Wright PJ, Braden AL, Wilt JA, Kraus SW. Internet pornography use and sexual motivation: a systematic review and integration. Ann Int Commun Assoc. 2019;43:117-55.

10. Paul B. Predicting internet pornography use and arousal: the role of individual difference variables. J Sex Res. 2009;46:344-57.

11. Magid V, Maclean MG, Colder CR. Differentiating between sensation seeking and impulsivity through their mediated relations with alcohol use and problems. Addict Behav. 2007;32:2046-61.

12. Birnbaum GE, Gillath O. Measuring subgoals of the sexual behavioral system: what is sex good for? J Soc Pers Relat. 2006;23:675-701.

13. Grubbs JB, Exline JJ. Trait entitlement: a cognitive-personality source of vulnerability to psychological distress. Psychol Bull. 2016;142:1204-26.

14. Brown CC, Durtschi JA, Carroll JS, Willoughby BJ. Understanding and predicting classes of college students who use pornography. Comput Hum Behav. 2017;66:114-21.

15. Baltazar A, Helm HW, Mcbride D, Hopkins G, Stevens JV. Internet pornography use in the context of external and internal religiosity. J Psychol Theol. 2010;38:32-40.

16. Peter J, Valkenburg PM. The use of sexually explicit internet material and its antecedents: a longitudinal comparison of adolescents and adults. Arch Sex Behav. 2011;40:1015-25.

17. Carvalheira A, Træen B, Stulhofer A. Masturbation and pornography use among coupled heterosexual men with decreased sexual desire: how many roles of masturbation? J Sex Marital Ther. 2015;41:626-35. 
18. Reid RC, Li DS, Gilliland R, Stein JA, Fong T. Reliability, validity, and psychometric development of the pornography consumption inventory in a sample of hypersexual men. J Sex Marital Ther. 2011;37:359-85.

19. Paul B, Shim JW. Gender, sexual affect, and motivations for internet pornography use. Int J Sex Health. 2008;20:187-99.

20. Castro-Calvo J, Ballester-Arnal R, Potenza MN, King DL, Billieux J. Does "forced abstinence" from gaming lead to pornography use? Insight from the April 2018 crash of Fortnite's servers. J Behav Addict. 2018;7:501-02.

21. Pornbub. Coronavirus insights. https://www.pornhub.com/ insights/corona-virus (2020).

22. Cacciamani GE, Bassi S, Sebben M, Marcer A, Russo GI, Cocci A, et al. Consulting "Dr. Google" for prostate cancer treatment options: a contemporary worldwide trend analysis. Eur Urol Oncol. 2020;3:481-8. https://doi.org/10.1016/j.euo.2019.07.002.

23. Russo GI, di Mauro M, Cocci A, Cacciamani G, Cimino S, Serefoglu EC, et al. Consulting "Dr Google" for sexual dysfunction: a contemporary worldwide trend analysis. Int J Impot Res. 2020;32:455-61. https://doi.org/10.1038/s41443-019-0203-2.

24. Nuti SV, Wayda B, Ranasinghe I, Wang S, Dreyer RP, Chen SI, et al. The use of Google Trends in health care research: a systematic review. PLoS ONE. 2014;9:e109583.

25. Schootman M, Toor A, Cavazos-Rehg P, Jeffe DB, McQueen A, Eberth J, et al. The utility of Google Trends data to examine interest in cancer screening. BMJ Open. 2015;5:e006678.

26. Google. Google Trends. www.google.com/trends.

27. JH. M https://surveillance.cancer.gov/joinpoint/Joinpoint_Help_4. 5.0.1.pdf.

28. Kim HJ, Fay MP, Feuer EJ, Midthune DN. Permutation tests for joinpoint regression with applications to cancer rates. Stat Med. 2000;19:335-51.

29. Kafka MP. Hypersexual disorder: a proposed diagnosis for DSMV. Arch Sex Behav. 2010;39:377-400.

30. Schenk CB. Pornography as a leisure behavior: an investigation of pornography use and leisure boredom. 2009. All Theses and Dissertations. 1976. https://scholarsarchive.byu.edu/etd/1976.

31. Rothman EF, Kaczmarsky C, Burke N, Jansen E, Baughman A. "Without Porn ... I Wouldn't Know Half the Things I Know Now": a qualitative study of pornography use among a sample of urban, low-income, black and hispanic youth. J Sex Res. 2015;52:736-46.
32. de Oliveira L, Carvalho J. The link between boredom and hypersexuality: a systematic review. J Sex Med. 2020;17:9941004. https://doi.org/10.1016/j.jsxm.2020.02.007.

33. Chaney M, Chang C. A trio of turmoil for internet sexually addicted men who have sex with men: boredom proneness, social connectedness, and dissociation. Sex Addict Compuls. 2005;12:3-18.

34. Lehmiller JJ. The psychology of human sexuality. 2nd ed. Oxford: Wiley-Blackwell; 2017.

35. Weber M, Aufenanger S, Dreier M, Quiring O, Reinecke L, Wölfling K, et al. Gender differences in escapist uses of sexually explicit internet material: results from a German probability sample. Sex Cult. 2018;22:1171-88.

36. Yoder VC, Virden TB, Amin K. Internet pornography and loneliness: an association? Sex Addict Compuls. 2005;12:19-44.

37. Goldenberg JL, McCoy SK, Pyszczynski T, Greenberg J, Solomon S. The body as a source of self-esteem: the effect of mortality salience on identification with one's body, interest in sex, and appearance monitoring. J Pers Soc Psychol. 2000;79:118-30.

38. Jacob L, Smith L, Butler L, Barnett Y, Grabovac I, McDermott D, et al. Challenges in the practice of sexual medicine in the time of COVID-19 in the United Kingdom. J Sex Med. 2020;17:1229-36.

39. Schiavi MC, Spina V, Zullo MA, Colagiovanni V, Luffarelli P, Rago R, et al. Love in the time of COVID-19: sexual function and quality of life analysis during the social distancing measures in a group of italian reproductive-age women. J Sex Med. 2020;17:1407-13

40. Li W, Li G, Xin C, Wang Y, Yang S. Challenges in the practice of sexual medicine in the time of COVID-19 in China. J Sex Med. 2020;17:1225-8. https://doi.org/10.1016/j.jsxm.2020.04.380

41. Ko NY, Lu WH, Chen YL, Li DJ, Chang YP, Wu CF, et al. Changes in sex life among people in Taiwan during the COVID19 pandemic: the roles of risk perception, general anxiety, and demographic characteristics. Int J Environ Res Public Health. 2020;17:E5822. https://doi.org/10.3390/ijerph17165822.

42. Kelley K, Musialowski D. Repeated exposure to sexually explicit stimuli: novelty, sex, and sexual attitudes. Arch Sex Behav. 1986;15:487-98.

43. Dutton DG, Aron AP. Some evidence for heightened sexual attraction under conditions of high anxiety. J Pers Soc Psychol. 1974;30:510-7. 